\title{
Parity Violation in ep scattering at JLab
}

\author{
P. A. Souder \\ Department of Physics, Syracuse University, Syracuse, NY13244
}

\begin{abstract}
We review the program of parity violation in the scattering of polarized electrons at JLab. Results are presented from recent experiments measuring the weak form factors, which in turn measure the contribution of strange quarks to the elastic form factors. In addition, we discuss the physics of parity violation in deep inelastic scattering, which will become possible with the upgrade of the JLab energy to $12 \mathrm{GeV}$.
\end{abstract}

Keywords: Parity violation, Form Factors, Structure Functions

PACS: $11.30 . \mathrm{Er}, 134.0 . \mathrm{Gp}, 13.60 . \mathrm{Hb}$

\section{INTRODUCTION}

By measuring the parity-violating asymmetry in the scattering of polarized electrons $A_{P V}=\left(\sigma_{\uparrow}-\sigma_{\downarrow}\right) /\left(\sigma_{\uparrow}+\sigma_{\downarrow}\right)$, we determine the electroweak interference between the photon and the $Z$ boson. From this information, we can extract weak amplitudes from electron scattering. The first experiment of this type was done by Prescott et al. [1], who in 1978 measured a $10^{-4}$ asymmetry in deep inelastic scattering, thereby establishing that the interactions of the $Z$ are parity violating.

Recently, there has been a large number of experimental results published on parity violation in electron scattering from SLAC, Mainz, Bates, and JLab. The asymmetries measured have been as small as $10^{-7}$. The goals of these experiments have been either to probe hadronic structure or to test the Standard Model [2].

\section{ELASTIC SCATTERING}

Elastic scattering from the proton, deuteron, and He targets is a classic example of using the weak interaction to probe hadronic structure [3,4]. Elastic scattering is determined by two functions of $Q^{2}, G_{E}$ and $G_{M}$. Both form factors have contributions from the various quarks, including $u, d$, and possibly $s$. The strangeness contribution to the form factors, possibly caused by a "kaon cloud" around the nucleon, can be differentiated from the larger $u$ and $d$ contributions by measuring the weak form factor.

Before this year, data on parity violation were consistent with the absence of significant strange contribution to the form factors. Then the A4 experiment at Mainz published a $2 \sigma$ effect at $Q^{2}=0.1(\mathrm{GeV} / \mathrm{c})^{2}$ [5]. With the publication of data from the HAPPEX $[6,7]$ and $G_{0}$ [8] collaborations at JLab, the significance of the world's data at $Q^{2}=0.1(\mathrm{GeV} / \mathrm{c})^{2}$ remains at about the $2 \sigma$ level. However, data from $G_{0}$, which covers a wide range of $Q^{2}$, indicates that the observed effect of strangeness in the range $Q^{2}=0.2-0.3(\mathrm{GeV} / \mathrm{c})^{2}$ is quite small. Thus strange form factors, if they are large, have 
a rapid $Q^{2}$ dependence. Further experiments are planned that will clarify this potentially exciting situation.

\section{DEEP INELASTIC SCATTERING}

In the limit of deep inelastic scattering (DIS), the parity-violating asymmetry is given by

$$
A^{P V}=Q^{2} \frac{G_{F}}{2 \sqrt{2} \pi \alpha}\left[a(x)+\frac{1-(1-y)^{2}}{1+(1-y)^{2}} b(x)\right],
$$

with $a(x)=\sum_{i} f_{i}(x) C_{1 i} Q_{i} / \sum_{i} f_{i}(x) Q_{i}^{2}$, and $b(x)=\sum_{i} f_{i}(x) C_{2 i} Q_{i} / \sum_{i} f_{i}(x) Q_{i}^{2}$. Here $f_{i}(x)$ is the probability of a parton with flavor $i$ having a fraction $x$ of the momentum of the nucleon. Also $Q_{i}$ is the electromagnetic charge and $C_{1 i}\left(C_{2} i\right)$ are the weak vector (axial vector) charges for the $i^{\text {th }}$ flavor. For an isoscalar target such as deuterium, we have

$$
a(x)=\frac{6}{5}\left[\left(C_{1 u}-\frac{1}{2} C_{1 d}\right)+\frac{2}{15} \frac{s(x)+\bar{s}(x)-c(x)-\bar{c}(x)}{D}\right]
$$

The key feature is that large $a(x)$ term is constant and independent of hadron structure at moderately large $x$ where the heavy quarks make small contributions. Thus the deuteron makes an ideal target for tests of the Standard Model[9].

For hydrogen targets, the $a(x)$ term is given by

$$
a(x) \approx \frac{3}{4}\left[\frac{6 C_{1 u} u(x)-3 C_{1 d} d(x)}{u(x)+\frac{1}{4} d(x)}\right] \sim\left[\frac{u(x)+0.912 d(x)}{u(x)+0.25 d(x)}\right]
$$

In this case the asymmetry depends on the ratio $d(x) / u(x)$, a quantity of considerable interest for very large $x[10,11,12,13]$.

\section{Possible Hadronic Corrections}

Since any interesting corrections to Eq. 1 are at most 5-10\%, the experiments must attain precision at the 1-2\% level. However, the surprising results of the NuTeV experiment [14] have led to a critical examination of whether all hadronic effects are understood to this high precision. We discuss below two possible effects which might be interesting in themselves, higher twist and charge symmetry.

\section{Higher Twist}

One interesting candidate for hadronic corrections are the higher twist (HT) effects, which might add $Q^{2}$ dependence to the asymmetry [15]:

$$
A^{P V}\left(x, Q^{2}\right)=A^{P V}(x)\left(1+C(x) / Q^{2}\right) .
$$


The size of the coefficients $C(x)$ is not known. However, the effect might be about the same size as the corresponding coefficients in the formula

$$
F_{2}\left(x, Q^{2}\right)=F_{2}(x)\left(1+D(x) / Q^{2}\right) .
$$

extracted from GLAP fits to structure function data $F_{2}\left(x, Q^{2}\right)$ [16]. The sizes of the $D(x)$ for $x<0.4$ are negligible for GLAP fits at the NNNLO level. However, the effects may be substantial and easy to measure for $x>0.5-0.6$. $A_{P V}$ makes an ideal laboratory for studying HT because the dominant $a(x)$ term is constant and requires no GLAP evolution.

\section{Charge Symmetry Violation}

One of the assumptions in deriving Eq. 1 is charge symmetry: $u^{p}=d^{n}$ and $d^{p}=u^{n}$. If we allow charge symmetry violation (CSV), new structure functions are required:

$$
\delta u(x)=u^{p}(x)-d^{n}(x) ; \delta d(x)=d^{p}(x)-u^{n}(x) .
$$

The effect of the CSV parameters on $R^{P W}$, the quantity measured by $\mathrm{NuTeV}$, is[17]:

$$
\frac{\delta R^{P W}}{R^{P W}} \sim 0.85 \frac{\delta u-\delta d}{u+d} .
$$

For parity, we have

$$
\frac{\delta A^{P V}}{A^{P V}}=0.28 \frac{\delta u-\delta d}{u+d} .
$$

Knowledge about $(\delta u-\delta d) /(u+d)$ is limited. Empirical limits from the MRST group allow CSV effects to be large enough to explain the NuTeV result[18].

The asymmetry $A^{P V}$ is less sensitive to CSV than is $R^{P W}$. However, for the NuTeV experiment with its large kinematic acceptance, the data is averaged over a large range in $x$. With the spectrometers at JLab, data may be obtained over a narrow kinematic range and in particular focus on the region of large $x$. Then if the CSV ratio in Eq. 2 is larger at high $x$, perhaps because of the falling values of the structure functions, measurements of $A P V$ at JLab might observe a significant effect. Any experimental demonstration of an $x$-dependent CSV would be extremely important.

\section{Required Apparatus}

A DIS point can be done with existing facilities at JLab at low $x$ and low $Q^{2}$. With the advent of the $12 \mathrm{GeV}$ upgrade and the SHMS spectrometer, additional points can be reached. Also, useful data with $W<2 \mathrm{GeV}$ can be obtained. However, to reach points with the highest $Q^{2}$ and $x$ values with sufficient statistics, a spectrometer with at least $50 \%$ acceptance in azimuth operating at angles of $20^{\circ}-40^{\circ}$ is required. We do not know how to achieve this goal economically with a conventional magnetic spectrometer. 
We suggest that a calorimeter such as the one used for A4 at Mainz might be more suitable. At the higher beam energies at JLab, the calorimeter must be shielded from photons and low energy pions originating in the target. Thus a sweeping magnet would be required. Such an apparatus will also be useful for many other physics topics, such as measurements of $A_{1}(x)$.

\section{Summary of the DIS Program}

With the proposed spectrometer, we can plan to measure $A_{P V}$ for hydrogen and deuterium with $<2 \%$ relative precision over the range $0.3<x<0.7$ with $Q^{2}$ varying by a factor of 2 for each point except at $x=0.7$. Unexpected variation of $A_{P V}$ for the deuteron with $Q^{2}$ or $x$ would be a signature for HT effects or CSV respectively. If these effects are demonstrated to be under control, we then can use deuterium as a test of the Standard Model and measure $d / u$ at high $x$ for hydrogen.

\section{ACKNOWLEDGMENTS}

I would like to thank A. Afanesev, D. Beck, P. Bosted, J. T. Londergan, K. Kumar, K. McFarland, F. Maas, Z. Meziani, W. Melnitchouk, M. J. Ramsey-Musolf, P. Reimer, A. Thomas, and M. Vanderhaeghen for useful discussions. This work is supported by the U. S. Department of Energy under Grant No. DE-FG020-84ER40146.

\section{REFERENCES}

1. C. Y. Prescott, et al., Phys. Lett. B 84, 524 (1979).

2. M. J. Musolf, T. W. Donnelly, J. Dubach, S. J. .. Pollock, S. Kowalski and E. J. Beise, Phys. Rept. 239, 1 (1994).

3. K. S. Kumar and P. A. Souder, Prog. Part. Nucl. Phys. 45, S333 (2000).

4. D. H. Beck and B. R. Holstein, Int. J. Mod. Phys. E 10, 1 (2001) [arXiv:hep-ph/0102053].

5. F. E. Maas et al., Phys. Rev. Lett. 94, 152001 (2005).

6. K. A. Aniol et al. [HAPPEX Collaboration], arXiv:nucl-ex/0506011.

7. K. A. Aniol et al. [HAPPEX Collaboration], arXiv:nucl-ex/0506010.

8. D. S. Armstrong et al. [G0 Collaboration], arXiv:nucl-ex/0506021.

9. A. Kurylov, M. J. Ramsey-Musolf and S. Su, Phys. Lett. B 582, 222 (2004).

10. W. Melnitchouk, I. R. Afnan, F. Bissey and A. W. Thomas, Phys. Rev. Lett. 84, 5455 (2000).

11. W. Melnitchouk and A. W. Thomas, Phys. Lett. B 377, 11 (1996).

12. S. I. Alekhin, Phys. Rev. D 63, 094022 (2001).

13. S. Kuhlmann et al., Phys. Lett. B 476, 297 (2000) .

14. G. P. Zeller et al. [NuTeV Collaboration], Phys. Rev. Lett. 88, 091802 (2002) [Erratum-ibid. 90, 239902 (2003)].

15. S. Brodsky, in "Proceedings from the JLab/Temple University HiX2000 Workshop,", 2000.

16. A. D. Martin, R. G. Roberts, W. J. Stirling and R. S. Thorne, Eur. Phys. J. C 35, 325 (2004).

17. J. T. Londergan and A. W. Thomas, Phys. Rev. D 67, 111901 (2003).

18. J. T. Londergan, Eur. Phys. J. A 24S2, 85 (2005). 